\title{
Spin-orbit angle in compact planetary systems perturbed by an inclined companion. Application to the 55 Cancri system
}

\author{
Gwenaël Boué $^{1}$ and Daniel C. Fabrycky ${ }^{2}$ \\ ${ }^{1}$ Sorbonne Universités, UPMC Univ Paris 06, UMR 8028, ASD-IMCCE, \\ Observatoire de Paris, F-75014 Paris, France \\ email: boue@imcce.fr \\ ${ }^{2}$ Department of Astronomy and Astrophysics, University of Chicago, \\ 5640 South Ellis Avenue, Chicago, IL 60637, USA \\ email: fabrycky@uchicago.edu
}

\begin{abstract}
The stellar spin orientation relative to the orbital planes of multiplanet systems are becoming accessible to observations. For example, 55 Cancri is a system composed of 5 planets orbiting a member of a stellar binary for which a projected obliquity of $72 \pm 12^{\circ}$ relative to the orbit of the innermost planet has been reported (Bourrier \& Hébrard 2014). This large obliquity has been attributed to the perturbation induced by the binary. Here we describe the secular evolution of similar systems and we discuss the case of the 55 Cancri system more deeply. We provide two different orbital configurations compatible with the currently available observations.
\end{abstract}

Keywords. celestial mechanics, methods: analytical, stars: planetary systems, stars: rotation, stars: binaries: general

\section{Introduction}

Consider a gravitational system composed of a central star, several planets on relatively tight orbits, and a perturbing body at a large semimajor axis. Any inclination of the outermost orbit relative to the inner ones would produce a long term precession of the planet orbital planes. Moreover, if the planets are close enough to each other, they all tilt in concert and their secular motion can be described as a solid rotation of the whole planet system (Innanen et al. 1997). In the following, this constraint is assumed to be fulfilled.

The goal of the present study is to determine the full range of spin-orbit angle that can be generated by the secular precession motion described above. By definition, the spin-orbit angle - also called the stellar obliquity or simply the obliquity - is the angle between the spin-axis of the central star and the normal of the planet plane. This specific problem has been analyzed extensively in Boué \& Fabrycky (2014). Here, the method is applied to 55 Cancri, a 5 planet system in a stellar binary.

This work is motivated by a recent measurement of the projected spin-orbit angle of 55 Cancri performed by Bourrier \& Hébrard (2014) using the Rossiter-McLaughlin effect. Their observations suggest a highly misaligned system with a projected obliquity $\lambda=72 \pm 12^{\circ}$. This measurement is, however, disputed by Lopez-Morales et al. (2014).

\section{Mathematical description of the problem}

The configuration of the system is uniquely defined by three unit vectors $\vec{s}, \vec{w}$, and $\vec{n}$ along the angular momentum of the stellar rotation, of the planet orbital motion, and 


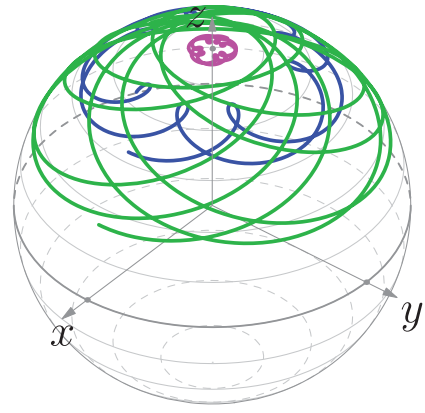

fixed frame

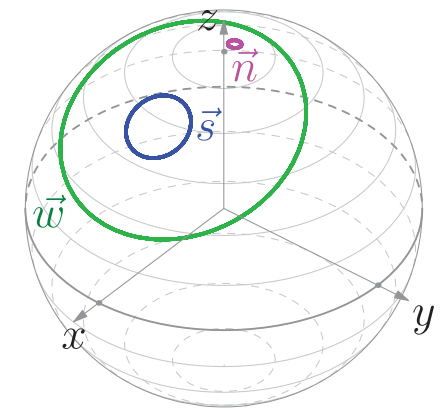

rotating frame

Figure 1. Typical solution of the equations (2.1) seen in a fixed reference frame (left) and in a rotating frame (right). The total angular momentum is aligned with the $z$-axis.

of the companion's orbit, respectively. Their motion is governed by the following secular equations (Boué \& Fabrycky 2014)

$$
\begin{aligned}
\frac{d \vec{s}}{d t} & =-\nu_{1}(\vec{s} \cdot \vec{w}) \vec{w} \times \vec{s}, \\
\frac{d \vec{w}}{d t} & =-\nu_{2}(\vec{s} \cdot \vec{w}) \vec{s} \times \vec{w}-\nu_{3}(\vec{n} \cdot \vec{w}) \vec{n} \times \vec{w}, \\
\frac{d \vec{n}}{d t} & =-\nu_{4}(\vec{n} \cdot \vec{w}) \vec{w} \times \vec{n} .
\end{aligned}
$$

Thus, the evolution is fully characterized by four secular frequencies $\nu_{1}, \nu_{2}, \nu_{3}$, and $\nu_{4}$, only. These frequencies represent the speed at which each vector precesses around the others. The interaction between $\vec{s}$ and $\vec{w}$ is due to the oblateness of the central star induced by its rotation. A similar interaction should exist between $\vec{s}$ and $\vec{n}$, but given its weakness, it is neglected.

The system described by the differential equations (2.1) belongs to a class of integrable three vector problems (Boué \& Laskar 2006, 2009). The general solution is a uniform rotation of the three vectors around the total angular momentum combined with periodic loops in the rotating frame (see figure 1).

\section{Amplitude of spin-orbit oscillations}

In the specific case where the perturber is a star, the binary's orbit possesses most of the total angular momentum. By consequence, its plane is practically invariant and the precession frequency $\nu_{4}$ is negligible with respect to the other frequencies. Furthermore, if we discard the timescale of the evolution, the three remaining frequencies can be normalized by an arbitrary constant so that the effective dimension of the parameter space becomes equal to two. Using barycentric coordinates $\left(\nu_{1} / \nu_{\text {tot }}, \nu_{2} / \nu_{\text {tot }}, \nu_{3} / \nu_{\text {tot }}\right)$, where $\nu_{\text {tot }}=\nu_{1}+\nu_{2}+\nu_{3}$, all systems with identical spin-orbit behavior but different timescales can be represented by a single point in a ternary diagram (Figure 2).

In this figure, close to the upper corner, as in the 55 Cancri case, the frequency $\nu_{1}$ is the highest, thus the evolution is dominated by the precession of the central star around the planet plane. Near the bottom left vertex, the strongest motion is the precession of the planets with respect to the equator of the star at the frequency $\nu_{2}$. Finally, in the vicinity of the bottom right corner where $\nu_{3}$ dominates, the evolution is mainly characterized by the precession of the planets around the binary's orbital plane. 


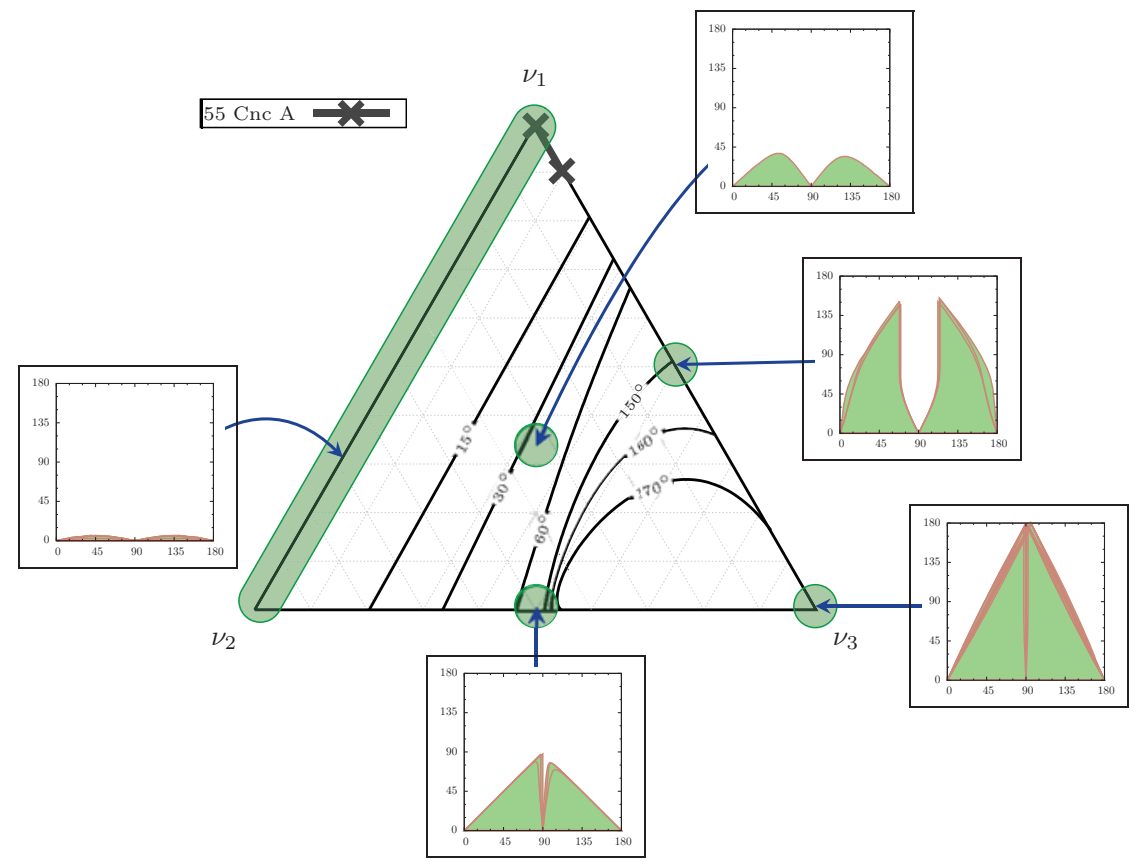

Figure 2. Ternary diagram with coordinates $\left(\nu_{1}, \nu_{2}, \nu_{3}\right)$. The system 55 Cancri is represented by a segment corresponding to the binary eccentricity ranging from 0 (top left end) to 0.95 (bottom right end). Any position in this diagram uniquely determines the behavior of the stellar obliquity. This value increases from a few degrees at the $\nu_{1}-\nu_{2}$ edge up to twice the initial inclination of the binary's orbit at the $\nu_{3}$ vertex of the triangle. Five insets detail the obliquity's full amplitude (vertical axis, in degrees) as a function of the binary's initial inclination (hortizontal axis, in degrees), the maximum of which is shown as level curves across the diagram.

As a result, all systems in the neighborhood of the $\nu_{1}-\nu_{2}$ edge are lead by a strong coupling between the central star and the planets. Hence, if the spin-orbit angle is initially small, it remains small. Conversely, close to the $\nu_{3}$ vertex the precession of the planets around the binary's orbit is too fast for the star to follow, thus the obliquity increases up to twice the initial inclination of the binary. The middle of the $\nu_{1}-\nu_{3}$ edge is particular because it corresponds to a secular spin-orbit resonance in the vicinity of a Cassini state. In this case, the maximal obliquity can exceed twice the initial inclination of the binary.

\section{Discussion}

Using the default values of Boué \& Fabrycky (2014), the 55 Cancri system ends up in a region of the parameters space where no significant spin-orbit misalignment is expected (Figure 2). Yet, these parameters are the same as those considered by Kaib et al. (2011) who inferred a most probable projected obliquity of about $60^{\circ}$, in very good agreement with Bourrier \& Hébrard (2014) observations. The apparent discrepancy among the two dynamical analyses is due to the spin-orbit coupling between the star and the planets which was not taken into account in Kaib et al. (2011). By consequence, $\nu_{1}$ and $\nu_{2}$ were forced to zero and the system was artificially shifted at the $\nu_{3}$ corner in Figure 2.

In order to match the Rossiter-McLaughlin observations, it is necessary to enhance the coupling between the planets and the companion. One solution is to increase the eccentricity of the binary above $e^{\prime} \gtrsim 0.987$ (Boué \& Fabrycky 2014). With this value, 
the system reaches the Cassini regime (middle of the $\nu_{1}-\nu_{3}$ edge in Figure 2) and the obliquity can grow by a large amount.

Another solution is to assume that planet $\mathrm{d}$ is the actual perturber of the inner four planets. Indeed, planet $\mathrm{d}$ is well separated from the inner ones; its inclination with respect to the plane of the sky is estimated to be $i_{d}=53 \pm 7^{\circ}$ (McArthur et al. 2004) while planet e's inclination is close to $i_{e}=90^{\circ}$ (Winn et al. 2011), implying that these two planets are mutually inclined $\dagger$; moreover, with this hypothesis the system falls in the pure orbital regime $\left(\nu_{3} \sim \nu_{4}\right) \gg\left(\nu_{1}, \nu_{2}\right)$ compatible with large spin-orbit angles (Boué \& Fabrycky 2014). Nevertheless, this scenario does not provide any hint to explain the origin of the required large inclination of planet $d$.

\section{Conclusion}

55 Cancri is a multiplanet system whose central star possesses a binary companion. This particular hierarchy can produce a misalignment of the stellar spin-axis with respect to the normal of the planets' plane. The secular evolution of such systems is uniquely determined by their position in a ternary diagram in which coordinates are three precession frequencies $\left(\nu_{1}, \nu_{2}, \nu_{3}\right)$. The 55 Cancri system is located in a region of the diagram with no excitation of the stellar obliquity in contradiction with recent Rossiter-McLaughlin measurements. Nevertheless, two different orbital configurations in agreement with the currently available observations are able to solve this issue. In the first one, the eccentricity of the stellar companion is set to a high value: $e^{\prime} \gtrsim 0.987$. In the second, the outermost planet takes the place of the perturber.

\section{References}

Boué, G. \& Fabrycky, D. C. 2014, ApJ, 789, 111

Boué, G. \& Laskar, J. 2006, Icarus, 185, 312

Boué, G. \& Laskar, J. 2009, Icarus, 201, 750

Bourrier, V. \& Hébrard, G. 2014, A\& A, 569, A65

Innanen, K. A., Zheng, J. Q., Mikkola, S., \& Valtonen, M. J. 1997, AJ, 113, 1915

Kaib, N. A., Raymond, S. N., \& Duncan, M. J. 2011, ApJ, 742, L24

Lopez-Morales, M., Triaud, A. H. M. J., Rodler, F., Dumusque, X., Buchhave, L. A., Harutyunyan, A., Hoyer, S., Alonso, R., Gillon, M., Kaib, N. A., Latham, D. W., Lovis, C., Pepe, F., Queloz, D., Raymond, S. N., Segransan, D., Waldmann, I. P., \& Udry, S. 2014, ApJ, 792, L31

McArthur, B. E., Endl, M., Cochran, W. D., Benedict, G. F., Fischer, D. A., Marcy, G. W., Butler, R. P., Naef, D., Mayor, M., Queloz, D., Udry, S., \& Harrison, T. E. 2004, ApJ, 614, L81

Nelson, B. E., Ford, E. B., Wright, J. T., Fischer, D. A., von Braun, K., Howard, A. W., Payne, M. J., \& Dindar, S. 2014, MNRAS, 441, 442

Rein, H. 2012, ArXiv, e-print:1211.7121

Winn, J. N., Matthews, J. M., Dawson, R. I., Fabrycky, D., Holman, M. J., Kallinger, T., Kuschnig, R., Sasselov, D., Dragomir, D., Guenther, D. B., Moffat, A. F. J., Rowe, J. F., Rucinski, S., \& Weiss, W. W. 2011, ApJ, 737, L18

$\dagger$ Nelson et al.. (2014) showed if the middle three planets are in the plane of the outermost, then Kozai cycles could destroy the innnermost planet; however, we suggest the middle three lie near the plane of the innermost. 